\title{
Focal set of curves in the Minkowski space near lightlike points
}

\author{
Ana Claudia Nabarro* and Andrea de Jesus Sacramento ${ }^{\dagger}$
}

\begin{abstract}
We study the geometry of curves in the Minkowski space and in the de Sitter space, specially at points where the tangent direction is lightlike (i.e. has length zero) called lightlike points of the curve. We define the focal sets of these curves and study the metric structure of them. At the lightlike points, the focal set is not defined. We use singularity theory techniques to carry out our study and investigate the focal set near lightlike points.
\end{abstract}

॥

\section{Introduction}

The study of submanifolds in Minkowski space is of interest in relativity theory. We believe that it is important to study the geometry of submanifolds in the Minkowski space with the induced metric degenerating at some points on the submanifolds. For example, any closed (compact without boundary) surfaces in the Minkowski 3-space has an non-empty locus of points where the metric is degenerate. (We observe that there are various studies in geometry on such submanifolds. For example, in [13] a Gauss-Bonnet type theorem is proven, and in [10], the problem of how to extend the Levi-Civita connection to the locus of degeneracy of the metric is considered). For this reason, some authors started to investigate the geometry of such submanifolds using the singularity theory. The first step was to study the cases of curves in the Minkowski plane [12] and of surfaces in the Minkowski 3-space [14]. In [14], the authors studied the caustics of surfaces in the Minkowski 3-space. Although the focal set of the surface is not defined at points where the metric is degenerate, the caustic is. The properties of

*Supported by FAPESP grant 2013/02794-4

†Supported by FAPESP grant 2010/20301-7

2010 Mathematics Subject classification 58K05, 53D10, 53B30.

Key Words and Phrases. Focal set, curves in the Minkowski space, curves in the de Sitter spaces, lightlike point, metric structure. 
the induced metric on the caustic are studied in [14]. Submanifolds in pseudo-spheres of the Minkowski space are also studied in several papers. In [5], Izumiya-Pei-Sano defined the hyperbolic Gauss indicatrix of a hypersurface in the Minkowski space model of the hyperbolic space. The work in [5] set the foundations of applications of singularity theory for the extrinsic geometry of submanifolds in the hyperbolic space. The extrinsic geometry of spacelike or timelike submanifolds in other pseudo-spheres of the Minkowski-space are investigated in subsequent papers.

In this paper, we study the geometry of curves in Minkowski 3-space and in pseudospheres $S_{1}^{2}$ and $S_{1}^{3}$. In order to do this, we study the families of distance square functions on the curves. We study their focal sets and the bifurcation set of the family of the distance square functions on these curves in order to investigate what happens near the lightlike points.

The paper is organised as follows. Section 2 addresses some preliminary results and notions that are used in the paper. We define an open and dense set of curves, such that the lightlike points of a curve are isolated. Besides, given a curve in this set, passing by lightlike points, the curve changes from spacelike to timelike.

We consider in $\$ 3$ spacelike and timelike curves $\gamma$ using the Frenet-Serret formulae. These formulae and the family of distance squared functions on $\gamma$ are the main tools in this section. Here we study the geometry and metric structure of the focal set of $\gamma$.

In Section 4, we study the bifurcation set of the family of distance squared functions on $\gamma$ in the neighborhood of lightlike points of the curve. In this case, we cannot parametrise the curve by arc length, therefore we cannot use the Frenet-Serret formulae as in $\$ 3$,

In $\$ 5$ and $₫ 6$, we consider curves, the focal sets and the bifurcation sets in the pseudo-spheres $S_{1}^{2}$ and $S_{1}^{3}$. We study the metric structures of these sets locally at lightlike points of $\gamma$.

\section{Preliminaries}

The Minkowski space $\mathbb{R}_{1}^{n+1}$ is the vector space $\mathbb{R}^{n+1}$ endowed with the pseudo-scalar product $\langle x, y\rangle=-x_{1} y_{1}+x_{2} y_{2}+\ldots+x_{n+1} y_{n+1}$, for any $x=\left(x_{1}, x_{2}, \ldots, x_{n+1}\right)$ and $y=\left(y_{1}, y_{2}, \ldots, y_{n+1}\right)$ in $\mathbb{R}^{n+1}$. We say that a non-zero vector $x \in \mathbb{R}_{1}^{n+1}$ is spacelike if $\langle x, x\rangle>0$, lightlike if $\langle x, x\rangle=0$ and timelike if $\langle x, x\rangle<0$. The norm of a vector $x \in \mathbb{R}_{1}^{n+1}$ is defined by $\|x\|=\sqrt{|\langle x, x\rangle|}$. This is an example of the Lorentzian metric. In $\mathbb{R}_{1}^{3}$, the pseudo vector product of $x=\left(x_{1}, x_{2}, x_{3}\right)$ and $y=\left(y_{1}, y_{2}, y_{3}\right)$ is:

$$
x \wedge y=\left|\begin{array}{ccc}
-e_{1} & e_{2} & e_{3} \\
x_{1} & x_{2} & x_{3} \\
y_{1} & y_{2} & y_{3}
\end{array}\right|,
$$

where $\left\{e_{1}, e_{2}, e_{3}\right\}$ is the standard basis of $\mathbb{R}^{3}$. For basic concepts and details of properties, see [11]. 
We have the following pseudo-spheres in $\mathbb{R}_{1}^{n+1}$ with centre 0 and radius $r>0$,

$$
\begin{gathered}
H^{n}(-r)=\left\{x \in \mathbb{R}_{1}^{n+1} \mid\langle x, x\rangle=-r^{2}\right\}, \text { called Hyperbolic } n \text {-space } \\
S_{1}^{n}(r)=\left\{x \in \mathbb{R}_{1}^{n+1} \mid\langle x, x\rangle=r^{2}\right\}, \text { called de Sitter } n \text {-space } \\
L C^{*}=\left\{x \in \mathbb{R}_{1}^{n+1} \backslash\{0\} \mid\langle x, x\rangle=0\right\}, \text { called Lightcone. }
\end{gathered}
$$

Instead of $S_{1}^{n}(1)$, we usually write $S_{1}^{n}$.

Let $\mathrm{V}$ be a vector subspace of $\mathbb{R}_{1}^{n+1}$. Then we say that $\mathrm{V}$ is timelike if and only if $\mathrm{V}$ has a timelike vector, spacelike if and only if every non-zero vector in $\mathrm{V}$ is spacelike, or lightlike otherwise. For a non-zero vector $v \in \mathbb{R}_{1}^{n+1}$ and a real number $c$, we define a hyperplane with normal $v$ by

$$
P(v, c)=\left\{x \in \mathbb{R}_{1}^{n+1} \mid\langle x, v\rangle=c\right\} .
$$

We call $P(v, c)$ a spacelike hyperplane, a timelike hyperplane or lightlike hyperplane if $v$ is timelike, spacelike or lightlike, respectively.

We consider embeddings $\gamma: I \rightarrow \mathbb{R}_{1}^{n}$, where $I$ is an open interval of $\mathbb{R}$. The set $\operatorname{Emb}\left(I, \mathbb{R}_{1}^{n}\right)$ of such embeddings is endowed with the Whitney $C^{\infty}$-topology. We say that a property is generic if it is satisfied by curves in a residual subset of $\operatorname{Emb}\left(I, \mathbb{R}_{1}^{n}\right)$.

We say that $\gamma$ is spacelike (resp. timelike) if $\gamma^{\prime}(t)$ is a spacelike (resp. timelike) vector for all $t \in I$. A point $\gamma(t)$ is called a lightlike point if $\gamma^{\prime}(t)$ is a lightlike vector.

As in [12] for plane curves, we define the subset $\Omega$ of $\operatorname{Emb}\left(I, \mathbb{R}_{1}^{n}\right)$ such that a curve $\gamma$ is in $\Omega$ if and only if $\left\langle\gamma^{\prime \prime}(t), \gamma^{\prime}(t)\right\rangle \neq 0$ whenever $\left\langle\gamma^{\prime}(t), \gamma^{\prime}(t)\right\rangle=0$. One can show, using Thom's transversality results (see for example [1], Chapter 9), that $\Omega$ is a residual subset of $\operatorname{Emb}\left(I, \mathbb{R}_{1}^{n}\right)$.

Proposition 2.1 Let $\gamma \in \Omega$. Then the lightlike points of $\gamma$ are isolated points.

Proof The proof is similar to the case $n=2$ given in [12].

We observe that if the curve $\gamma \in \Omega$ then at a lightlike point $\gamma\left(t_{0}\right)$, the curve changes from a spacelike curve to a timelike curve or vice-versa.

To study the local properties of $\gamma$ at $\gamma\left(t_{0}\right)$, we use the germ $\gamma: \mathbb{R}, t_{0} \rightarrow \mathbb{R}_{1}^{3}$ of $\gamma$ at $t_{0}$. The family of distance squared functions $f: I \times \mathbb{R}_{1}^{3} \rightarrow \mathbb{R}$ on $\gamma$ is given by

$$
f(t, v)=\langle\gamma(t)-v, \gamma(t)-v\rangle
$$

We denote by $f_{v}: I \rightarrow \mathbb{R}$ the function $f_{v}(t)=f(t, v)$, for any fixed $v \in \mathbb{R}_{1}^{3}$.

The distance squared function $f_{v}$ has singularity of type $A_{k}$ at $t_{0}$ if the derivatives $\left(f_{v}\right)^{(p)}\left(t_{0}\right)=0$ for all $1 \leq p \leq k$, and $\left(f_{v}\right)^{(k+1)}\left(t_{0}\right) \neq 0$. We also say that $f_{v}$ has singularity of type $A_{\geq k}$ at $t_{0}$ if $\left(f_{v}\right)^{(p)}\left(t_{0}\right)=0$ for all $1 \leq p \leq k$. This is valid including if $\gamma\left(t_{0}\right)$ is a lightlike point of the curve. Now let $F: \mathbb{R}_{1}^{3} \rightarrow \mathbb{R}$ be a submersion and $\gamma: I \rightarrow \mathbb{R}_{1}^{3}$ be a regular curve. We say that $\gamma$ and $F^{-1}(0)$ have contact of order $k$ or 
$k$-point contact at $t=t_{0}$ if the function $g(t)=F \circ \gamma(t)$ satisfies $g\left(t_{0}\right)=g^{\prime}\left(t_{0}\right)=\cdots=$ $g^{(k)}\left(t_{0}\right)=0$ and $g^{(k+1)}\left(t_{0}\right) \neq 0$, i.e, if $g$ has singularity $A_{k}$ at $t_{0}$. Then the singularity type of $f_{v}$ at $t_{0}$ measures the contact of $\gamma$ at $\gamma\left(t_{0}\right)$ with the pseudo-sphere of centre $v$ and radius $\left\|\gamma\left(t_{0}\right)-v\right\|$. The type of pseudo-sphere is determined by the sign of $\left\langle\gamma\left(t_{0}\right)-v, \gamma\left(t_{0}\right)-v\right\rangle$. For a generic curve in $\mathbb{R}_{1}^{3}, f_{v}$ has local singularities of type $A_{1}$, $A_{2}, A_{3}$ or $A_{4}$ (see [9]), and the singularities $A_{4}$ occur at isolated points of the curve. If $f_{v_{0}}$ has an $A_{k}$-singularity $(k=2,3,4)$ at $t_{0}$, then $f$ is a $(p)$-versal unfolding of $f_{v_{0}}$ [9]. The bifurcation set of $f$ is given by

$$
\mathfrak{B i f}(f)=\left\{v \in \mathbb{R}_{1}^{3} \mid f_{v}^{\prime}(t)=f_{v}^{\prime \prime}(t)=0 \text { in }(t, v) \text { for some } t\right\},
$$

i.e., the directions where $f_{v}$ at $t$ has a degenerate (non-stable) singularity, that is, the singularity is of type $A_{\geq 2}$. It is defined even when the point is a lightlike point of $\gamma$.

The focal set of $\gamma$, for spacelike or timelike curves, is the locus of centres of pseudospheres that has at least a 2-point contact with the curve. Therefore, the $\mathfrak{B i f}(f)$ and the focal set of $\gamma$ coincide for spacelike and timelike curves.

We have a fundamental result of the unfolding theory:

Theorem 2.2 [1] Let $G:\left(\mathbb{R} \times \mathbb{R}^{3},\left(t_{0}, v_{0}\right)\right) \rightarrow \mathbb{R}$ be a 3-parameter unfolding of $g(t)$ which has an $A_{k}$-singularity at $t_{0}$. Suppose that $G$ is a $(p)$-versal unfolding, then $\mathfrak{B i f}(G)$ is locally diffeomorphic to

(a) $\mathbb{R}^{2}$, if $k=2$;

(b) cuspidal edge $C \times \mathbb{R}$, if $k=3$;

(c) swallowtail $S W$, if $k=4$,

where $C=\left\{\left(x_{1}, x_{2}\right) \mid x_{1}^{2}=x_{2}^{3}\right\}$ is the ordinary cusp and $S W=\left\{\left(x_{1}, x_{2}, x_{3}\right) \mid x_{1}=\right.$ $\left.3 u^{4}+u^{2} v, x_{2}=4 u^{3}+2 u v, x_{3}=v\right\}$ is the swallowtail (see [1] for figure of the $S W$ surface).

\section{The focal sets of spacelike and timelike curves}

Let $\gamma: I \rightarrow \mathbb{R}_{1}^{3}$ be a spacelike or a timelike curve and suppose that it is parametrised by arc length. This is possible because $\gamma$ has no lightlike points.

In this section, we remember the Frenet-Serret formulae of $\gamma$ and we find the parametrisation of their focal surfaces. Furthermore, we study the metric structure of these focal surfaces.

We denote by $t$ the unit tangent vector to $\gamma$. Let $n$ be the unit normal vector to $\gamma$ given by $\gamma^{\prime \prime}(s)=k(s) n(s)$, where $k(s)=\left\|\gamma^{\prime \prime}(s)\right\|$ is defined as being the curvature of $\gamma$ at $s$, and $b(s)=t(s) \wedge n(s)$ the unit binormal vector to $\gamma(s)$. Then, we have the orthonormal basis $\{t(s), n(s), b(s)\}$ of $\mathbb{R}_{1}^{3}$ along $\gamma$. Using exactly the same arguments 
as the case for a curve in an Euclidian 3-space, we have the following Frenet-Serret formulae (see [8], [4]):

$$
\left\{\begin{aligned}
t^{\prime}(s) & =k(s) n(s) \\
n^{\prime}(s) & =-\varepsilon(\gamma(s)) \delta(\gamma(s)) k(s) t(s)+\varepsilon(\gamma(s)) \tau(s) b(s), \\
b^{\prime}(s) & =\tau(s) n(s)
\end{aligned}\right.
$$

with $\tau(s)$ being the torsion of $\gamma$ at $s, \varepsilon(\gamma(s))=\operatorname{sign}(t(s)), \delta(\gamma(s))=\operatorname{sign}(n(s))$, where $\operatorname{sign}(v)$ is 1 if the vector $v$ is spacelike or -1 if the vector $v$ is timelike. We call them, $\varepsilon$ and $\delta$ for short.

Observe that if $\gamma$ is a spacelike or a timelike curve and $k(s)=0$ for some $s \in I$, then $f_{v}^{\prime \prime}(s)=\varepsilon(\gamma(s)) \neq 0$ and there is no singularity $A_{\geq 2}$. Now if $\tau(s)=0$ for some $s \in I$, then generically $f_{v}^{(3)}(s)=-\varepsilon(\gamma(s)) k^{\prime}(s) \neq 0$, that is, there is no singularity $A_{\geq 3}$. This is the reason for which $k(s) \neq 0$ and $\tau(s) \neq 0$ in the following proposition.

Proposition 3.1 [9] Let $\gamma: I \rightarrow \mathbb{R}_{1}^{3}$ be a spacelike or a timelike curve parametrised by arc length, with $k(s) \neq 0$ and $\tau(s) \neq 0$. Then

(1) $f_{v}^{\prime}\left(s_{0}\right)=0$ if and only if there exist $\lambda, \mu \in \mathbb{R}$ such that $\gamma\left(s_{0}\right)-v=\lambda n\left(s_{0}\right)+\mu b\left(s_{0}\right)$.

(2) $f_{v}^{\prime}\left(s_{0}\right)=f_{v}^{\prime \prime}\left(s_{0}\right)=0$ if and only if $v=\gamma\left(s_{0}\right)+\frac{\varepsilon\left(\gamma\left(s_{0}\right)\right)}{\delta\left(\gamma\left(s_{0}\right)\right) k\left(s_{0}\right)} n\left(s_{0}\right)+\mu b\left(s_{0}\right)$ for some $\mu \in \mathbb{R}$.

(3) $f_{v}^{\prime}\left(s_{0}\right)=f_{v}^{\prime \prime}\left(s_{0}\right)=f_{v}^{(3)}\left(s_{0}\right)=0$ if and only if

$$
v=\gamma\left(s_{0}\right)+\frac{\varepsilon\left(\gamma\left(s_{0}\right)\right)}{\delta\left(\gamma\left(s_{0}\right)\right) k\left(s_{0}\right)} n\left(s_{0}\right)+\frac{k^{\prime}\left(s_{0}\right)}{\varepsilon\left(\gamma\left(s_{0}\right)\right) \delta\left(\gamma\left(s_{0}\right)\right) k^{2}\left(s_{0}\right) \tau\left(s_{0}\right)} b\left(s_{0}\right)
$$

Thus, for a spacelike or timelike curve $\gamma$ parametrised by arc length with $k(s) \neq 0$, we have that the focal surface of $\gamma$ is given by

$$
\mathfrak{B}(s, \mu)=\gamma(s)+\frac{\varepsilon(\gamma(s))}{\delta(\gamma(s)) k(s)} n(s)+\mu b(s),
$$

with $\mu \in \mathbb{R}$. The cuspidal curve of the focal surface is given by

$$
\mathfrak{B}(s)=\gamma(s)+\frac{\varepsilon(\gamma(s))}{\delta(\gamma(s)) k(s)} n(s)+\mu(s) b(s),
$$

with $\mu(s)=\frac{k^{\prime}(s)}{\varepsilon(\gamma(s)) \delta(\gamma(s)) k^{2}(s) \tau(s)}$, that is, where the distance squared function has singularity $A_{\geq 3}$. We denote the cuspidal curve $\mathfrak{B}(s)$ by $\mathcal{C}$.

We observe that the focal surface is a developable surface (for more details see [3] ). 
Proposition 3.2 Let $\gamma$ be a connected timelike curve, then $\gamma$ does not intersect its focal surface.

Proof Suppose that $\gamma$ is timelike and intersects its focal surface, then there exists $s_{1}, s_{2} \in I$ with $s_{1} \neq s_{2}$ (for simplicity suppose that $s_{2}<s_{1}$ ) such that,

$$
\gamma\left(s_{1}\right)-\frac{1}{k\left(s_{1}\right)} n\left(s_{1}\right)+\mu b\left(s_{1}\right)=\gamma\left(s_{2}\right)
$$

Consider the function $g:\left[s_{2}, s_{1}\right] \rightarrow \mathbb{R}$ given by $g(s)=\left\langle\gamma(s), \gamma^{\prime}\left(s_{1}\right)\right\rangle-\left\langle\gamma\left(s_{1}\right), \gamma^{\prime}\left(s_{1}\right)\right\rangle$. Thus $g\left(s_{1}\right)=g\left(s_{2}\right)=0$ and therefore by the Rolle's theorem exists $s_{3} \in\left(s_{2}, s_{1}\right)$ such that $g^{\prime}\left(s_{3}\right)=0$. Since $g^{\prime}\left(s_{3}\right)=\left\langle\gamma^{\prime}\left(s_{1}\right), \gamma^{\prime}\left(s_{3}\right)\right\rangle$ we have that $\gamma^{\prime}\left(s_{3}\right)$ belongs to a plane generated by $n\left(s_{1}\right)$ and $b\left(s_{1}\right)$. But this is a contradiction because this plane is spacelike and contains $\gamma^{\prime}\left(s_{3}\right)$ that is timelike. Therefore, $\gamma$ does not intersect its focal surface.

Remark 3.3 If $\gamma$ is spacelike and $\gamma$ intersects its focal surface, then generically its occurs at isolated points because this is given by a generic transversality condition.

To study the metric structure of the focal surface $\mathfrak{B}$, we need some concepts. A spacelike surface is a surface for which the tangent plane, at any point, is a spacelike plane (i.e., consists only of spacelike vectors). A timelike surface is a surface for which the tangent plane, at any point, is a timelike plane (i.e., consists of spacelike, timelike and lightlike vectors).

The pseudo scalar product in $\mathbb{R}_{1}^{3}$ induces a metric on the focal surface $\mathfrak{B}$ that may be degenerated at some points of $\mathfrak{B}$. This means that the tangent planes to $\mathfrak{B}$ are lightlike at these points. We label the locus of such points the Locus of Degeneracy and we denote it by $L D$ (see [14] for Locus of Degeneracy of caustics of surfaces in $\mathbb{R}_{1}^{3}$ ). The LD of $\mathfrak{B}$ may be empty (Theorem 3.4, (d)) or a smooth curve (Proposition 4.3) that splits the focal surface $\mathfrak{B}$ locally into a Riemannian (where the tangent planes are spacelike) and a Lorentzian region (where the tangent planes are timelike). It is interesting to study what happens at points where the metric is degenerate and explain the changes in the geometry from a Riemannian region to a Lorentzian region of the submanifold (see \$4 ). Furthermore, the focal surface can have points where the tangent plane is not defined.

Consider the focal surface of a spacelike or a timelike curve $\gamma$, that is,

$$
\mathfrak{B}(s, \mu)=\gamma(s)+\frac{\varepsilon(\gamma(s))}{\delta(\gamma(s)) k(s)} n(s)+\mu b(s), \quad \mu \in \mathbb{R} .
$$

Observe that $\mathfrak{B}_{s}=\frac{\partial \mathfrak{B}}{\partial s}(s, \mu)$ is parallel to $\mathfrak{B}_{\mu}=\frac{\partial \mathfrak{B}}{\partial \mu}(s, \mu)$ if and only if

$$
\mu(s)=\frac{k^{\prime}(s)}{\varepsilon(\gamma(s)) \delta(\gamma(s)) k^{2}(s) \tau(s)},
$$


and $\mathfrak{B}(s, \mu(s))$ is the parametrisation of the curve where $f_{v}$ has singularities of type $A_{\geq 3}$, that is the cuspidal curve $\mathcal{C}$.

Supposing

$$
\mu(s) \neq \frac{k^{\prime}(s)}{\varepsilon(\gamma(s)) \delta(\gamma(s)) k^{2}(s) \tau(s)},
$$

then $\mathfrak{B}_{s}$ and $\mathfrak{B}_{\mu}$ generate the tangent planes of the surface $\mathfrak{B}$, and for $v=\lambda_{1} \mathfrak{B}_{s}+\lambda_{2} \mathfrak{B}_{\mu}$,

$$
\langle v, v\rangle=\lambda_{1}^{2}\left(\frac{\tau^{2}}{k^{2}}\langle b, b\rangle+\frac{k^{\prime 2} \delta}{k^{4}}-2 \frac{\varepsilon k^{\prime} \mu \tau}{k^{2}}+\mu^{2} \tau^{2} \delta\right)+2 \lambda_{1} \lambda_{2}\left(\frac{\tau}{\delta k}\langle b, b\rangle\right)+\lambda_{2}^{2}\langle b, b\rangle .
$$

We use this expression in the following theorem.

The above calculations show the item (a) of the next result, that is, the tangent plane of the focal surface is not defined only at the points of the cuspidal curve $\mathcal{C}$ given by Equation (2).

Theorem 3.4 (a) Only at the points of the cuspidal curve $\mathcal{C}$, the tangent planes of the focal surface are not defined.

Away from the cuspidal curve $\mathcal{C}$ :

(b) the focal surface of a timelike generic curve is spacelike;

(c) the focal surface of a spacelike generic curve is timelike;

(d) if the curve is spacelike and timelike, then the LD set of the focal surface is empty.

Proof (b) Let $\gamma$ be a timelike curve, then $n(s)$ and $b(s)$ are spacelike. Therefore, $v=$ $\lambda_{1} \mathfrak{B}_{s}+\lambda_{2} \mathfrak{B}_{\mu}$ are vectors on the tangent plane and

$$
\langle v, v\rangle=\lambda_{1}^{2}\left(\left(\frac{k^{\prime}}{k^{2}}+\mu \tau\right)^{2}+\frac{\tau^{2}}{k^{2}}\right)(s)+2 \lambda_{1} \lambda_{2}\left(\frac{\tau}{k}\right)(s)+\lambda_{2}^{2} .
$$

Making $\langle v, v\rangle=0$, we can think in the above equation as a quadratic equation of $\lambda_{1}$, thus $\Delta=-4 \lambda_{2}^{2}\left(\frac{k^{\prime}}{k^{2}}+\mu \tau\right)^{2}(s) \leq 0$.

Since $\mu(s) \neq-\frac{k^{\prime}}{k^{2} \tau}(s)$ at the regular points of the focal surface, then $\Delta=0 \Leftrightarrow \lambda_{2}=0$. Replacing $\lambda_{2}=0$ in $(*)$, we have a lightlike direction if $\tau(s)=0$ and $k^{\prime}(s)=0$, but we are supposing $\tau(s) \neq 0$ (see Proposition [3.1), so $\Delta<0$. Thus, we do not have lightlike directions in this plane, and therefore the tangent planes are spacelike.

(c) Let $\gamma$ be a spacelike curve, then we have two cases: $n(s)$ timelike and b(s) spacelike or $n(s)$ spacelike and $b(s)$ timelike.

In the case where $n(s)$ is timelike and $b(s)$ is spacelike, we have

$$
\langle v, v\rangle=\lambda_{1}^{2}\left(\frac{\tau^{2}}{k^{2}}-\left(\frac{k^{\prime}}{k^{2}}+\mu \tau\right)^{2}\right)(s)-2 \lambda_{1} \lambda_{2}\left(\frac{\tau}{k}\right)(s)+\lambda_{2}^{2} . \quad(* *)
$$


Similar to (b), we obtain $\Delta \geq 0$ and at the regular points $\Delta=0 \Leftrightarrow \lambda_{2}=0$. Replacing $\lambda_{2}=0$ in $(* *)$ we have a lightlike direction if $\left(\frac{\tau^{2}}{k^{2}}-\left(\frac{k^{\prime}}{k^{2}}+\mu \tau\right)^{2}\right)(s)=0$, i.e., $\mu(s)=\mu_{1}(s)=$

$$
\begin{aligned}
\left(\frac{1}{k}-\frac{k^{\prime}}{k^{2} \tau}\right)(s) \text { or } \mu(s) & =\mu_{2}(s)=\left(-\frac{1}{k}-\frac{k^{\prime}}{k^{2} \tau}\right)(s) \text {. Then } \\
& \mathfrak{B}_{s}\left(s, \mu_{1}(s)\right)=\frac{\tau(s)}{k(s)}(n(s)-b(s)) \text { and } \mathfrak{B}_{s}\left(s, \mu_{2}(s)\right)=-\frac{\tau(s)}{k(s)}(n(s)+b(s))
\end{aligned}
$$

are linearly independent lightlike vectors and $\mathfrak{B}_{\mu}\left(s, \mu_{1}(s)\right)=\mathfrak{B}_{\mu}\left(s, \mu_{2}(s)\right)=b(s)$. On the other hand, we have

$$
\mathfrak{B}_{s}\left(s, \mu_{2}(s)\right)=-\mathfrak{B}_{s}\left(s, \mu_{1}(s)\right)-\frac{2 \tau(s)}{k(s)} \mathfrak{B}_{\mu}\left(s, \mu_{1}(s)\right),
$$

i.e., $\mathfrak{B}_{s}\left(s, \mu_{2}(s)\right)$ belongs to the plane generated by $\mathfrak{B}_{s}\left(s, \mu_{1}(s)\right)$ and $\mathfrak{B}_{\mu}\left(s, \mu_{1}(s)\right)$. Similarly, the vector $\mathfrak{B}_{s}\left(s, \mu_{1}(s)\right)$ is in the plane generated by $\mathfrak{B}_{s}\left(s, \mu_{2}(s)\right)$ and $\mathfrak{B}_{\mu}\left(s, \mu_{2}(s)\right)$. Therefore if $\lambda_{2}=0$, the tangent planes at $\mathfrak{B}\left(s, \mu_{1}(s)\right)$ and at $\mathfrak{B}\left(s, \mu_{2}(s)\right)$ will have two lightlike directions and then these planes will be timelike. Thus, we conclude that at all points of the focal surface, the tangent plane is timelike.

When $n(s)$ is spacelike and $b(s)$ is timelike, it follows that at all points of the focal surface, the tangent plane is timelike, because $\mathfrak{B}_{\mu}(s, \mu)=b(s)$ where $b(s)$ is timelike.

(d) The LD set is empty and it is a consequence of (a), (b) and (c).

\section{The focal set near lightlike points}

So far, we have studied what is happening to the focal surface of a spacelike or a timelike curve. The focal set is not defined at the lightlike points of the curve, but at these points, the bifurcation set of the family of distance squared functions on the curve is defined. Furthermore, the focal set is contained in the bifurcation set and they coincide if the curve is spacelike or timelike. Consider a curve with lightlike points. As the curve is in $\Omega$, these points are isolated (Proposition 2.1) and the curve changes from spacelike to timelike at these points. We can think of the bifurcation set as a form of pass from the focal set of the spacelike side of the curve to the focal set of the timelike side of the curve. Our main goal in this section is then to understand this passage by studying the geometry of the bifurcation set near the lightlike point of the curve. The principal result in this section is given by Theorem 4.3 ,

To study the bifurcation set near lightlike points $\gamma\left(t_{0}\right)$ of $\gamma$, we cannot parametrise the curve by the arc length since $\left\langle\gamma^{\prime}\left(t_{0}\right), \gamma^{\prime}\left(t_{0}\right)\right\rangle=0$. We consider then a smooth and regular curve $\gamma: I \rightarrow \mathbb{R}_{1}^{3}$ not parametrised by the arc length. The distance squared function is given by $f_{v}(t)=\langle\gamma(t)-v, \gamma(t)-v\rangle$. Thus

$$
\frac{1}{2} f_{v}^{\prime}(t)=\left\langle\gamma(t)-v, \gamma^{\prime}(t)\right\rangle
$$


It follows that $f_{v}$ is singular at $t$ if and only if $\left\langle\gamma(t)-v, \gamma^{\prime}(t)\right\rangle=0$, equivalently, $\gamma(t)-v=$ $\mu N(t)+\lambda B(t)$, where $N(t)$ and $B(t)$ are vectors that generate the normal plane to the vector $\gamma^{\prime}(t)$. (This condition includes the lightlike points.)

Differentiating again we obtain

$$
\frac{1}{2} f_{v}^{\prime \prime}(t)=\left\langle\gamma(t)-v, \gamma^{\prime \prime}(t)\right\rangle+\left\langle\gamma^{\prime}(t), \gamma^{\prime}(t)\right\rangle
$$

The singularity of $f_{v}$ is degenerate if and only if $f_{v}^{\prime}(t)=f_{v}^{\prime \prime}(t)=0$, equivalently, $\gamma(t)-v=$ $\mu N(t)+\lambda B(t)$ and

$$
\mu\left\langle N(t), \gamma^{\prime \prime}(t)\right\rangle+\lambda\left\langle B(t), \gamma^{\prime \prime}(t)\right\rangle+\left\langle\gamma^{\prime}(t), \gamma^{\prime}(t)\right\rangle=0 .
$$

It follows that the bifurcation set of $f$ is given by

$$
\mathfrak{B i f}(f)=\{\gamma(t)-\mu N(t)-\lambda B(t) \mid(\mu, \lambda) \text { is solution of (3i) }\} .
$$

Away from the isolated lightlike points of $\gamma$, the bifurcation set is precisely the focal surface of the spacelike and timelike components of $\gamma$ studied in Section 3 ,

Now our aim is to study the general expression (4) of the bifurcation set of the family of distance squared functions on the curve, to analyse what is happening with this surface when the curve $\gamma$ has lightlike points. We remember that since $\gamma \in \Omega$, then near a lightlike point, $\gamma$ changes from a spacelike curve to a timelike curve.

Taking $N(t)=\gamma^{\prime}(t) \wedge \gamma^{\prime \prime}(t)$ and $B(t)=\gamma^{\prime}(t) \wedge\left(\gamma^{\prime}(t) \wedge \gamma^{\prime \prime}(t)\right)$ and replacing in (3) and (4), we have that the bifurcation set of $f$ can be written as

$$
\mathfrak{B i f}(f)=\left\{\gamma(t)-\mu N(t)-\frac{\left\langle\gamma^{\prime}(t), \gamma^{\prime}(t)\right\rangle}{\left\langle\gamma^{\prime}(t) \wedge \gamma^{\prime \prime}(t), \gamma^{\prime}(t) \wedge \gamma^{\prime \prime}(t)\right\rangle} B(t) \mid \mu \in \mathbb{R}\right\} .
$$

In short, we will denote the map that defines $\mathfrak{B i f}(f)$ as $\mathfrak{B}(t, \mu)$, and the $\mathfrak{B} \mathfrak{i f}(f)$ set also as $\mathfrak{B}$.

Since $\gamma \in \Omega$, at a lightlike point $\gamma\left(t_{0}\right)$ of $\gamma$, the vector $N\left(t_{0}\right)=\gamma^{\prime}\left(t_{0}\right) \wedge \gamma^{\prime \prime}\left(t_{0}\right)$ is not lightlike, thus the bifurcation set above is well defined in the neighborhood of $t_{0}$. Furthermore, $B\left(t_{0}\right)$ is parallel to $\gamma^{\prime}\left(t_{0}\right)$, and the vectors $N\left(t_{0}\right)$ and $B\left(t_{0}\right)$ generate the normal plane to $\gamma^{\prime}\left(t_{0}\right)$. In this case as $\left\langle\gamma^{\prime}\left(t_{0}\right), \gamma^{\prime}\left(t_{0}\right)\right\rangle=0$, then $\gamma^{\prime}\left(t_{0}\right)$ is contained in this normal plane, which is a situation totally different from the Euclidian case.

Given a curve $\gamma$ with lightlike points $\gamma\left(t_{0}\right)$, in the next result, we prove which types of singularities can occur if $v=\mathfrak{B}\left(t_{0}, \mu\right)$ for the distance squared function $f_{v}$. These are the only points of the bifurcation set that are not in the focal surfaces of the spacelike and timelike parts of the curve.

Proposition 4.1 Let $\gamma \in \Omega$. If $\gamma\left(t_{0}\right)$ is the lightlike point of $\gamma$ and $v=\mathfrak{B}\left(t_{0}, \mu\right)$ then the distance squared function $f_{v}$ has $A_{2}$-singularity except if $\mu_{0}=\frac{-3\left\langle\gamma^{\prime}\left(t_{0}\right), \gamma^{\prime \prime}\left(t_{0}\right)\right\rangle}{\left\langle\gamma^{\prime}\left(t_{0}\right) \wedge \gamma^{\prime \prime}\left(t_{0}\right), \gamma^{\prime \prime \prime}\left(t_{0}\right)\right\rangle}$, where $f_{v_{0}}$ has $A_{\geq 3}$-singularity for $v_{0}=\mathfrak{B}\left(t_{0}, \mu_{0}\right)$. 
Proof Consider $f_{v}(t)=\langle\gamma(t)-v, \gamma(t)-v\rangle$ the distance squared function on $\gamma$. Then $f_{v}^{(3)}(t)=6\left\langle\gamma^{\prime}(t), \gamma^{\prime \prime}(t)\right\rangle+2\left\langle\gamma(t)-v, \gamma^{\prime \prime \prime}(t)\right\rangle$, i.e.,

$$
f_{v}^{(3)}(t)=6\left\langle\gamma^{\prime}(t), \gamma^{\prime \prime}(t)\right\rangle+2\left\langle\lambda(t) \gamma^{\prime}(t) \wedge\left(\gamma^{\prime}(t) \wedge \gamma^{\prime \prime}(t)\right)+\mu \gamma^{\prime}(t) \wedge \gamma^{\prime \prime}(t), \gamma^{\prime \prime \prime}(t)\right\rangle .
$$

Therefore, at lightlike point $\gamma\left(t_{0}\right), f_{v}^{(3)}\left(t_{0}\right)=6\left\langle\gamma^{\prime}\left(t_{0}\right), \gamma^{\prime \prime}\left(t_{0}\right)\right\rangle+2\left\langle\mu \gamma^{\prime}\left(t_{0}\right) \wedge \gamma^{\prime \prime}\left(t_{0}\right), \gamma^{\prime \prime \prime}\left(t_{0}\right)\right\rangle$. Remember that we are supposing as in Section 3 that the torsion is non zero at $t_{0}$ and therefore $\left\langle\gamma^{\prime}\left(t_{0}\right) \wedge \gamma^{\prime \prime}\left(t_{0}\right), \gamma^{\prime \prime \prime}\left(t_{0}\right)\right\rangle \neq 0$. Then we have $f_{v}^{(3)}\left(t_{0}\right)=0$ if and only if $\mu=$ $\frac{-3\left\langle\gamma^{\prime}\left(t_{0}\right), \gamma^{\prime \prime}\left(t_{0}\right)\right\rangle}{\left\langle\gamma^{\prime}\left(t_{0}\right) \wedge \gamma^{\prime \prime}\left(t_{0}\right), \gamma^{\prime \prime \prime}\left(t_{0}\right)\right\rangle} \neq 0$, because $\gamma \in \Omega$.

We analyse the curve $\mathfrak{B}\left(t_{0}, \mu\right), \mu \in \mathbb{R}$, of the surface $\mathfrak{B}$, that is a curve that split the focal surface of the spacelike side of $\gamma$ of the focal surface of the timelike side of $\gamma$.

Proposition 4.2 (a) Let $\gamma: I \rightarrow \mathbb{R}_{1}^{3}$ be a regular curve with $\gamma\left(t_{0}\right)$ a lightlike point of $\gamma$. On the points of the curve $\mathfrak{B}\left(t_{0}, \mu\right), \mu \in \mathbb{R}$, surface $\mathfrak{B}$ has degenerate tangent plane except for $\mu_{0}=\frac{-3\left\langle\gamma^{\prime}\left(t_{0}\right), \gamma^{\prime \prime}\left(t_{0}\right)\right\rangle}{\left\langle\gamma^{\prime}\left(t_{0}\right) \wedge \gamma^{\prime \prime}\left(t_{0}\right), \gamma^{\prime \prime \prime}\left(t_{0}\right)\right\rangle}$, where the tangent plane is not defined. Thus, the LD set of $\mathfrak{B}$ is $\mathfrak{B}\left(t_{0}, \mu\right)$ with $\mu \neq \mu_{0}$.

(b) The curve $\mathfrak{B}\left(t_{0}, \mu\right), \mu \in \mathbb{R}$, intersects the cuspidal curve $\mathcal{C}$ when $\mu=\mu_{0}$.

Proof (a) We have

$\frac{\partial \mathfrak{B}}{\partial t}(t, \mu)=\gamma^{\prime}(t)-\mu\left(\gamma^{\prime}(t) \wedge \gamma^{\prime \prime \prime}(t)\right)-\lambda^{\prime}(t)\left(\gamma^{\prime}(t) \wedge\left(\gamma^{\prime}(t) \wedge \gamma^{\prime \prime}(t)\right)\right)-\lambda(t)\left(\gamma^{\prime}(t) \wedge\left(\gamma^{\prime}(t) \wedge \gamma^{\prime \prime}(t)\right)\right)^{\prime}$ $\frac{\partial \mathfrak{B}}{\partial \mu}(t, \mu)=-\left(\gamma^{\prime}(t) \wedge \gamma^{\prime \prime}(t)\right)$, where

$\lambda^{\prime}(t)=\frac{2\left\langle\gamma^{\prime}(t), \gamma^{\prime \prime}(t)\right\rangle\left\langle\gamma^{\prime}(t) \wedge \gamma^{\prime \prime}(t), \gamma^{\prime}(t) \wedge \gamma^{\prime \prime}(t)\right\rangle-2\left\langle\gamma^{\prime}(t), \gamma^{\prime}(t)\right\rangle\left\langle\gamma^{\prime}(t) \wedge \gamma^{\prime \prime}(t), \gamma^{\prime}(t) \wedge \gamma^{\prime \prime \prime}(t)\right\rangle}{\left(\left\langle\gamma^{\prime}(t) \wedge \gamma^{\prime \prime}(t), \gamma^{\prime}(t) \wedge \gamma^{\prime \prime}(t)\right\rangle\right)^{2}}$

Then $\frac{\partial \mathfrak{B}}{\partial t}\left(t_{0}, \mu\right)=3 \gamma^{\prime}\left(t_{0}\right)-\mu\left(\gamma^{\prime}\left(t_{0}\right) \wedge \gamma^{\prime \prime \prime}\left(t_{0}\right)\right)$ and $\frac{\partial \mathfrak{B}}{\partial \mu}\left(t_{0}, \mu\right)=-\left(\gamma^{\prime}\left(t_{0}\right) \wedge \gamma^{\prime \prime}\left(t_{0}\right)\right)$, and therefore

$$
\frac{\partial \mathfrak{B}}{\partial t}\left(t_{0}, \mu\right) \wedge \frac{\partial \mathfrak{B}}{\partial \mu}\left(t_{0}, \mu\right)=\left(3\left\langle\gamma^{\prime}\left(t_{0}\right), \gamma^{\prime \prime}\left(t_{0}\right)\right\rangle+\mu\left\langle\gamma^{\prime}\left(t_{0}\right) \wedge \gamma^{\prime \prime}\left(t_{0}\right), \gamma^{\prime \prime \prime}\left(t_{0}\right)\right\rangle\right) \gamma^{\prime}\left(t_{0}\right) . \quad \text { As the }
$$

torsion is non zero at $t_{0}$, we have that the vectors $\gamma^{\prime}\left(t_{0}\right), \gamma^{\prime \prime}\left(t_{0}\right)$ and $\gamma^{\prime \prime \prime}\left(t_{0}\right)$ are linearly independent and then $\frac{\partial \mathfrak{B}}{\partial t}\left(t_{0}, \mu\right)$ and $\frac{\partial \mathfrak{B}}{\partial \mu}\left(t_{0}, \mu\right)$ are linearly dependent if and only if $\mu=$ $\frac{-3\left\langle\gamma^{\prime}\left(t_{0}\right), \gamma^{\prime \prime}\left(t_{0}\right)\right\rangle}{\left\langle\gamma^{\prime}\left(t_{0}\right) \wedge \gamma^{\prime \prime}\left(t_{0}\right), \gamma^{\prime \prime \prime}\left(t_{0}\right)\right\rangle}$, which we call $\mu_{0}$.

Furthermore, using Theorem 3.4, we have that the focal surface of the spacelike side of the curve is timelike and that the focal surface of the timelike side of the curve is spacelike. Thus, the LD is contained in $\mathfrak{B}\left(t_{0}, \mu\right)$.

Supposing $\mu \neq \mu_{0}$, the vectors of the tangent planes at the points of the curve $\mathfrak{B}\left(t_{0}, \mu\right)$ are given by:

$$
v=\lambda_{1}\left(3 \gamma^{\prime}\left(t_{0}\right)-\mu\left(\gamma^{\prime}\left(t_{0}\right) \wedge \gamma^{\prime \prime \prime}\left(t_{0}\right)\right)\right)-\lambda_{2}\left(\gamma^{\prime}\left(t_{0}\right) \wedge \gamma^{\prime \prime}\left(t_{0}\right)\right)
$$


Then

$$
\begin{aligned}
& \langle v, v\rangle=\lambda_{1}^{2} \mu^{2}\left\langle\gamma^{\prime}\left(t_{0}\right) \wedge \gamma^{\prime \prime \prime}\left(t_{0}\right), \gamma^{\prime}\left(t_{0}\right) \wedge \gamma^{\prime \prime \prime}\left(t_{0}\right)\right\rangle+2 \lambda_{1} \lambda_{2} \mu\left\langle\gamma^{\prime}\left(t_{0}\right) \wedge \gamma^{\prime \prime \prime}\left(t_{0}\right), \gamma^{\prime}\left(t_{0}\right) \wedge \gamma^{\prime \prime}\left(t_{0}\right)\right\rangle+ \\
& \lambda_{2}^{2}\left\langle\gamma^{\prime}\left(t_{0}\right) \wedge \gamma^{\prime \prime}\left(t_{0}\right), \gamma^{\prime}\left(t_{0}\right) \wedge \gamma^{\prime \prime}\left(t_{0}\right)\right\rangle .
\end{aligned}
$$

Making $\langle v, v\rangle=0$ and thinking of the above equation as a quadratic equation of $\lambda_{2}$, we have $\Delta=0$. Therefore, each tangent plane has a unique lightlike direction, given by

$$
\left(\lambda_{1}, \lambda_{2}\right)=\left(\lambda_{1},-\lambda_{1} \frac{\mu\left\langle\gamma^{\prime}\left(t_{0}\right) \wedge \gamma^{\prime \prime \prime}\left(t_{0}\right), \gamma^{\prime}\left(t_{0}\right) \wedge \gamma^{\prime \prime}\left(t_{0}\right)\right\rangle}{\left\langle\gamma^{\prime}\left(t_{0}\right) \wedge \gamma^{\prime \prime}\left(t_{0}\right), \gamma^{\prime}\left(t_{0}\right) \wedge \gamma^{\prime \prime}\left(t_{0}\right)\right\rangle}\right)
$$

with $\lambda_{1} \neq 0$. Thus, the induced metric on these planes is degenerate and the curve $\mathfrak{B}\left(t_{0}, \mu\right)$, with $\mu \neq \mu_{0}$, is the $L D$ of the surface $\mathfrak{B}$. (Observe that the denominator is different from zero because $\gamma \in \Omega$.)

(b) The proof of this case follows from Proposition 4.1, where we have that $f_{v_{0}}$ has singularity $A_{\geq 3}$ for $v_{0}=\mathfrak{B}\left(t_{0}, \mu_{0}\right)$, and the cuspidal curve is precisely given by $v^{\prime} s$ where $f_{v}$ has singularity $A_{\geq 3}$.

We prove below that the surface $\mathfrak{B}$ intersects the curve $\gamma$ at the lightlike points and we study the geometric behavior of $\mathfrak{B}$ at the neighborhood of these points.

Theorem 4.3 Let $\gamma \in \Omega$, with $\gamma\left(t_{0}\right)$ lightlike point, and let $\mathfrak{B}$ be the bifurcation set of the family of distance squared functions on $\gamma$. Then

(1) the surface $\mathfrak{B}$ intersects the curve $\gamma$ locally only at the lightlike point $\gamma\left(t_{0}\right)$.

(2) the surface $\mathfrak{B}$ is regular at $\gamma\left(t_{0}\right)$.

(3) the tangent line to the curve at $\gamma\left(t_{0}\right)$ is contained in the tangent plane to $\mathfrak{B}$ at such a point, that is, the unique lightlike direction of the tangent plane of $\mathfrak{B}$ at $\gamma\left(t_{0}\right)$ is the direction of the tangent line of $\gamma$ at $\gamma\left(t_{0}\right)$.

(4) the $L D$ set of the surface $\mathfrak{B}$ is a normal line to the curve passing through $\gamma\left(t_{0}\right)$ and splits the focal surface into a Riemannian and a Lorentzian region.

Proof (1) Since $\gamma \in \Omega$, we have that at a lightlike point $\gamma\left(t_{0}\right)$,

$$
\frac{\left\langle\gamma^{\prime}\left(t_{0}\right), \gamma^{\prime}\left(t_{0}\right)\right\rangle}{\left\langle\gamma^{\prime}\left(t_{0}\right) \wedge \gamma^{\prime \prime}\left(t_{0}\right), \gamma^{\prime}\left(t_{0}\right) \wedge \gamma^{\prime \prime}\left(t_{0}\right)\right\rangle}=0
$$

Then $\mathfrak{B}\left(t_{0}, 0\right)=\gamma\left(t_{0}\right)$. Locally this intersection occurs only at the lightlike points because of Proposition 3.2 and Remark 3.3.

(2) From Proposition 4.1. we have that at $\gamma\left(t_{0}\right)=\mathfrak{B}\left(t_{0}, 0\right)=v_{0}, f_{v_{0}}$ has only singularity of type $A_{2}$. Thus, by Theorem [2.2, surface $\mathfrak{B}$ is locally regular at this point.

(3) Observe that $\gamma^{\prime}\left(t_{0}\right)$ belongs to the tangent plane of the surface at $\gamma\left(t_{0}\right)$, which is generated by $\frac{\partial \mathfrak{B}}{\partial t}\left(t_{0}, 0\right)=3 \gamma^{\prime}\left(t_{0}\right)$ and $\frac{\partial \mathfrak{B}}{\partial \mu}\left(t_{0}, 0\right)=-\left(\gamma^{\prime}\left(t_{0}\right) \wedge \gamma^{\prime \prime}\left(t_{0}\right)\right)$. 
Furthermore, the vectors of the tangent plane to the surface at $\gamma\left(t_{0}\right)$, are given by:

$$
v=3 \lambda_{1} \gamma^{\prime}\left(t_{0}\right)-\lambda_{2}\left(\gamma^{\prime}\left(t_{0}\right) \wedge \gamma^{\prime \prime}\left(t_{0}\right)\right),
$$

where $\lambda_{1}, \lambda_{2} \in \mathbb{R}$, and $\langle v, v\rangle=\lambda_{2}^{2}\left\langle\gamma^{\prime}\left(t_{0}\right), \gamma^{\prime \prime}\left(t_{0}\right)\right\rangle^{2} \geq 0$. Thus $\gamma^{\prime}\left(t_{0}\right)$ is the unique lightlike direction of the tangent plane, i.e. the tangent plane at $\gamma\left(t_{0}\right)$ is lightlike.

(4) As $\mathfrak{B}\left(t_{0}, \mu\right)=\gamma\left(t_{0}\right)-\mu N\left(t_{0}\right)$, then this normal line of $\gamma$ is contained in the focal surface. By Proposition $4.2(\mathrm{a})$, the $L D$ is $\mathfrak{B}\left(t_{0}, \mu\right)$ except when $\mu_{0}=\frac{-3\left\langle\gamma^{\prime}\left(t_{0}\right), \gamma^{\prime \prime}\left(t_{0}\right)\right\rangle}{\left\langle\gamma^{\prime}\left(t_{0}\right) \wedge \gamma^{\prime \prime}\left(t_{0}\right), \gamma^{\prime \prime \prime}\left(t_{0}\right)\right\rangle}$, which is different from zero because $\gamma \in \Omega$. Therefore, near the $\left(t_{0}, 0\right)$, i.e, near the $\mathfrak{B}\left(t_{0}, 0\right)=\gamma\left(t_{0}\right)$, the induced metric along this normal line is degenerate. For this, it is enough to take a neighborhood of $\left(t_{0}, 0\right)$ that does not contain $\mu_{0}$.

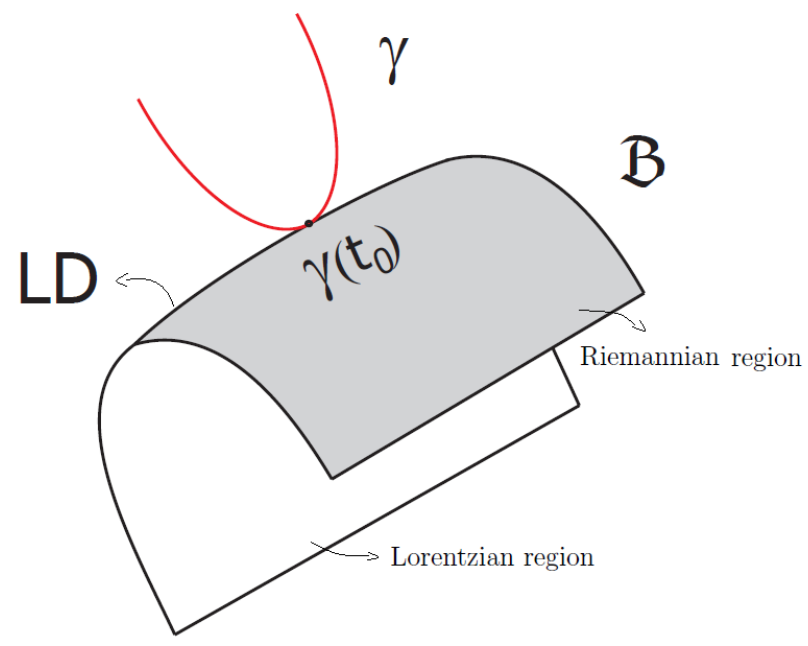

Figure 1: Metric structure of the focal surface locally at a lightlike point of $\gamma$.

Observe that the cuspidal curve $\mathcal{C}$ intersects the curve $\mathfrak{B}\left(t_{0}, \mu\right)$ at $\mathfrak{B}\left(t_{0}, \mu_{0}\right)$ (Proposition 4.2 (b)), i.e., away from lightlike points where $\mu=0$. In this case, the local configuration of the bifurcation set at $\mathfrak{B}\left(t_{0}, \mu_{0}\right)=v_{0}$ is as in Figure 2, if $f_{v_{0}}$ has singularity $A_{3}$ at $t_{0}$. 


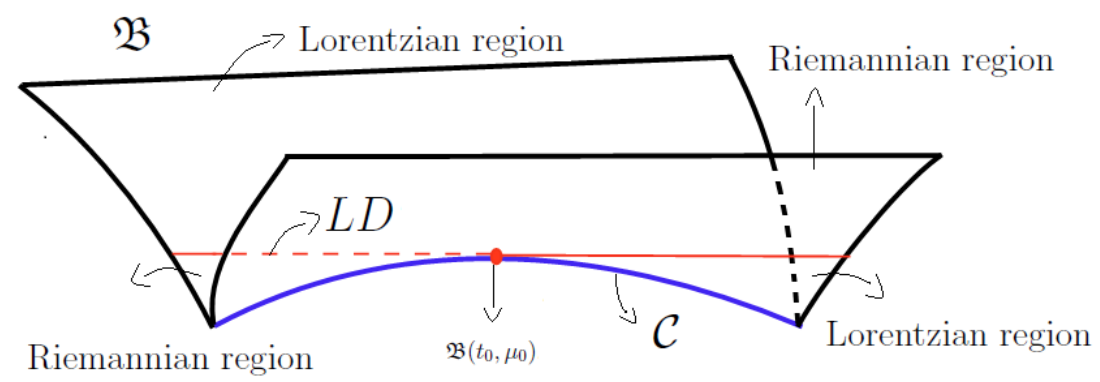

Figure 2: Example of a metric structure of a surface $\mathfrak{B}$ locally at no lightlike point $\mathfrak{B}\left(t_{0}, \mu_{0}\right)$.

In the case where $f_{v_{0}}$ has singularity $A_{4}$ at $t_{0}$, for $\mathfrak{B}\left(t_{0}, \mu_{0}\right)=v_{0}$, the curve $L D$ intersects the cuspidal curve at the singular point of $\mathcal{C}$.

\section{$5 \quad$ Focal set of curves in $S_{1}^{2}$}

In this section, we consider curves in the de Sitter space $S_{1}^{2} \subset \mathbb{R}_{1}^{3}$ and their focal sets also in $S_{1}^{2}$, which we call the spherical focal curve. To obtain the results, we have Section 4 as a motivation.

Let $\gamma: I \rightarrow S_{1}^{2}$ be a spacelike or a timelike smooth and regular curve in $S_{1}^{2}$ parametrised by the arc length. For this curve, consider the orthonormal basis $\left\{\gamma(s), t(s)=\gamma^{\prime}(s), n(s)=\right.$ $\gamma(s) \wedge t(s)\}$ of $\mathbb{R}_{1}^{3}$ along $\gamma$. By standard arguments, we have the following Frenet-Serret type formulae:

$$
\left\{\begin{aligned}
\gamma^{\prime}(s) & =t(s) \\
t^{\prime}(s) & =-\varepsilon(\gamma(s)) \gamma(s)+\delta(\gamma(s)) k_{g}(s) n(s), \\
n^{\prime}(s) & =-\varepsilon(\gamma(s)) k_{g}(s) t(s)
\end{aligned}\right.
$$

where $\varepsilon(\gamma(s))=\operatorname{sign}(t(s)), \delta(\gamma(s))=\operatorname{sign}(n(s))$ and $k_{g}(s)=\left\langle\gamma^{\prime \prime}(s), n(s)\right\rangle$ is the geodesic curvature of $\gamma$ at $s$.

Consider the family of distance squared functions $f: I \times S_{1}^{2} \rightarrow \mathbb{R}$ on $\gamma$, given by

$$
f(s, v)=\langle\gamma(s)-v, \gamma(s)-v\rangle,
$$

and $f_{v}: I \rightarrow \mathbb{R}$ given by $f_{v}(s)=f(s, v)$, for some $v \in S_{1}^{2}$ fixed.

The spherical bifurcation set of $f$ is given by

$$
\mathfrak{B i f}(f)=\left\{v \in S_{1}^{2} \mid f_{v}^{\prime}(s)=f_{v}^{\prime \prime}(s)=0 \text { in }(s, v) \text { for some } s\right\},
$$

i.e, the directions where the singularity of $f$ at $s$ is at least $A_{2}$. The spherical focal curve of $\gamma$ is given by the spherical bifurcation set of $f$. Furthermore, the spherical focal curve is the intersection of the focal surface in $\mathbb{R}_{1}^{3}$ with the de Sitter space $S_{1}^{2}$. Observe that since 
$-\frac{1}{2} f_{v}(s)=\langle\gamma(s), v\rangle-1$ if $v \in S_{1}^{2}$, then the singularities of the distance squared function and of the height function are the same. Therefore, the evolutes of a curve $\gamma$ in $S_{1}^{2}$, coincide with the spherical focal curve of $\gamma$. In [7], the authors study the evolutes of hyperbolic plane curves, that is, a curve in $H^{2}(-1)$ and these evolutes also coincide with the bifurcation set in $H^{2}(-1)$.

For a spacelike or a timelike curve $\gamma$ parametrised by the arc length with $k_{g}(s) \neq 0$, we have that the spherical focal curve of $\gamma$ is given by

$$
\alpha^{ \pm}(s)= \pm \frac{k_{g}(s)}{\sqrt{k_{g}^{2}(s)+\delta(\gamma(s))}} \gamma(s) \pm \frac{\varepsilon(\gamma(s))}{\sqrt{k_{g}^{2}(s)+\delta(\gamma(s))}} n(s) .
$$

Remark 5.1 To define the spherical focal curve, we must have $k_{g}^{2}(s)+\delta(\gamma(s))>0$. Then in the case that $\gamma$ is spacelike, we must have $k_{g}(s)<-1$ or $k_{g}(s)>1$ and in the case that $\gamma$ is timelike, the spherical focal curve is always defined. Furthermore, as $\alpha^{-}(s)=-\alpha^{+}(s)$ we work only with $\alpha^{+}(s)$.

Consider $\mathcal{C}$ the cuspidal curve of the focal surface of $\gamma$ in $\mathbb{R}_{1}^{3}$, as in Section 3 , Then, we have the next result.

Proposition 5.2 The singular points of the spherical focal curve of $\gamma$ are given by $S_{1}^{2} \cap \mathcal{C}$.

Proof Observe that $f_{v}$ has singularity $A_{\geq 3}$ at $s_{0}$ if and only if $k_{g}^{\prime}\left(s_{0}\right)=0$, equivalently $\alpha^{+}\left(s_{0}\right)$ (and $\alpha^{-}\left(s_{0}\right)$ ) is the singular point of the spherical focal curve, because

$$
\left(\alpha^{+}\right)^{\prime}(s)=\frac{\delta(\gamma(s)) k_{g}^{\prime}(s)}{\left(k_{g}^{2}(s)+\delta(\gamma(s))\right) \sqrt{k_{g}^{2}(s)+\delta(\gamma(s))}} \gamma(s)-\frac{\varepsilon(\gamma(s)) k_{g}(s) k_{g}^{\prime}(s)}{\left(k_{g}^{2}(s)+\delta(\gamma(s))\right) \sqrt{k_{g}^{2}(s)+\delta(\gamma(s))}} n(s) .
$$

In the next proposition, we study the metric structure of the spherical focal curve of a spacelike curve and of a timelike curve.

Proposition 5.3 Away from the singular points,

(a) the spherical focal curve of a spacelike curve is timelike;

(b) the spherical focal curve of a timelike curve is spacelike.

Proof (a) Away from the singular points of $\alpha^{+}$, i.e., where $k_{g}^{\prime}(s) \neq 0$ we have that $\alpha^{+}$is a timelike curve because

$$
\left\langle\left(\alpha^{+}\right)^{\prime}(s),\left(\alpha^{+}\right)^{\prime}(s)\right\rangle=\frac{-\left(k_{g}^{\prime}\right)^{2}(s)}{\left(k_{g}^{2}(s)-1\right)^{2}}<0 .
$$

(b) This proof is analogous to the case (a). 
We want to know what is happening at the lightlike points of a curve $\gamma$. For this, let us find an expression of the bifurcation set near a lightlike point of $\gamma$. Here we cannot consider $\gamma: I \rightarrow S_{1}^{2}$ parametrised by the arc length and the focal set is not defined at the lightlike point.

Let $\gamma(t)$ and $N(t)=\gamma(t) \wedge \gamma^{\prime}(t)$ be the vectors that generate the normal plane to the vector $\gamma^{\prime}(t)$ and consider the family of distance squared functions $f: I \times S_{1}^{2} \rightarrow \mathbb{R}$ on $\gamma$. By definition, we have that the bifurcation set of $f$ is given by

$\mathfrak{B i f}(f)=\left\{ \pm \sqrt{1+\mu^{2}\left\langle\gamma^{\prime}(t), \gamma^{\prime}(t)\right\rangle} \gamma(t)+\mu N(t) \mid \mu\right.$ is the solution of the equation $\left.\left(1^{ \pm}\right)\right\}$,

where $\mu\left\langle\gamma(t) \wedge \gamma^{\prime}(t), \gamma^{\prime \prime}(t)\right\rangle \pm \sqrt{1+\mu^{2}\left\langle\gamma^{\prime}(t), \gamma^{\prime}(t)\right\rangle}\left\langle\gamma(t), \gamma^{\prime \prime}(t)\right\rangle=0$.

Remark 5.4 Let $\gamma \in \Omega$ such that $\gamma\left(t_{0}\right)$ is a lightlike point of $\gamma$. In the next result, we use that $\left\langle\gamma\left(t_{0}\right) \wedge \gamma^{\prime}\left(t_{0}\right), \gamma^{\prime \prime}\left(t_{0}\right)\right\rangle \neq 0$. Indeed, if $\left\langle\gamma\left(t_{0}\right) \wedge \gamma^{\prime}\left(t_{0}\right), \gamma^{\prime \prime}\left(t_{0}\right)\right\rangle=0$ then exist $a, b \in \mathbb{R}$ with $a^{2}+b^{2} \neq 0$ such that $\gamma^{\prime \prime}\left(t_{0}\right)=a \gamma\left(t_{0}\right)+b \gamma^{\prime}\left(t_{0}\right)$, because $\gamma\left(t_{0}\right)$ and $\gamma^{\prime}\left(t_{0}\right)$ are vectors linearly independent. By supposing $a \neq 0$ and $b=0$, then $\left\langle\gamma^{\prime \prime}\left(t_{0}\right), \gamma\left(t_{0}\right)\right\rangle=a \neq 0$, that is $a$ contradiction, since $\left\langle\gamma(t), \gamma^{\prime \prime}(t)\right\rangle=-\left\langle\gamma^{\prime}(t), \gamma^{\prime}(t)\right\rangle$. Now suppose that $a=0$ and $b \neq 0$, then $\left\langle\gamma^{\prime \prime}\left(t_{0}\right), \gamma^{\prime}\left(t_{0}\right)\right\rangle=b\left\langle\gamma^{\prime}\left(t_{0}\right), \gamma^{\prime}\left(t_{0}\right)\right\rangle=0$, that is a contradiction, because $\gamma \in \Omega$. For $a \neq 0$ and $b \neq 0$, we get the same contradictions. Therefore, $\left\langle\gamma\left(t_{0}\right) \wedge \gamma^{\prime}\left(t_{0}\right), \gamma^{\prime \prime}\left(t_{0}\right)\right\rangle \neq 0$.

Solving the equation $\left(1^{+}\right)$and using the fact that $\left\langle\gamma(t), \gamma^{\prime \prime}(t)\right\rangle=-\left\langle\gamma^{\prime}(t), \gamma^{\prime}(t)\right\rangle$, it follows that the solutions are $\mu(t)$ or $-\mu(t)$ where

$$
\mu(t)=\frac{\left\langle\gamma^{\prime}(t), \gamma^{\prime}(t)\right\rangle}{\sqrt{\left\langle\gamma(t) \wedge \gamma^{\prime}(t), \gamma^{\prime \prime}(t)\right\rangle^{2}-\left\langle\gamma^{\prime}(t), \gamma^{\prime}(t)\right\rangle^{3}}} .
$$

Observe that in the neighborhood of $t_{0}$, the term inside of the root of the denominator is greater than zero, because of Remark $5.4\left\langle\gamma\left(t_{0}\right) \wedge \gamma^{\prime}\left(t_{0}\right), \gamma^{\prime \prime}\left(t_{0}\right)\right\rangle \neq 0$.

If $\left\langle\gamma\left(t_{0}\right) \wedge \gamma^{\prime}\left(t_{0}\right), \gamma^{\prime \prime}\left(t_{0}\right)\right\rangle>0$ then $\mu(t)$ is the solution of $\left(1^{+}\right)$and $-\mu(t)$ is the solution of $\left(1^{-}\right)$. If $\left\langle\gamma\left(t_{0}\right) \wedge \gamma^{\prime}\left(t_{0}\right), \gamma^{\prime \prime}\left(t_{0}\right)\right\rangle<0$ then $-\mu(t)$ is the solution of $\left(1^{+}\right)$and $\mu(t)$ is the solution of $\left(1^{-}\right)$. Therefore, we have that $\alpha^{+}(t)$ is a smooth curve and we can rewrite $\alpha^{+}(t)$ as

$$
\begin{gathered}
\sqrt{1+\mu^{2}(t)\left\langle\gamma^{\prime}(t), \gamma^{\prime}(t)\right\rangle} \gamma(t)+\mu(t) N(t) \text { if }\left\langle\gamma\left(t_{0}\right) \wedge \gamma^{\prime}\left(t_{0}\right), \gamma^{\prime \prime}\left(t_{0}\right)\right\rangle>0 \text { or } \\
\sqrt{1+\mu^{2}(t)\left\langle\gamma^{\prime}(t), \gamma^{\prime}(t)\right\rangle} \gamma(t)-\mu(t) N(t) \text { if }\left\langle\gamma\left(t_{0}\right) \wedge \gamma^{\prime}\left(t_{0}\right), \gamma^{\prime \prime}\left(t_{0}\right)\right\rangle<0 .
\end{gathered}
$$

The above bifurcation set is contained in $S_{1}^{2}$ and then we have a spherical curve of $\gamma$ given by $\mathfrak{B i f}(f)=\alpha^{+} \cup \alpha^{-}$, where $\alpha^{+}$and $\alpha^{-}$are symmetric.

Proposition 5.5 The spherical curve $\alpha^{+}$is a smooth curve that intersects the curve $\gamma$ at the lightlike points of $\gamma$. The curve $\alpha^{-}$does not intersect the curve $\gamma$, but it has the same geometry of $\alpha^{+}$, by symmetry.

Proof Let $\gamma\left(t_{0}\right)$ be a lightlike point of $\gamma$. The parametrisation of the spherical curve $\alpha^{+}$locally at $t_{0}$, is given as above and a proof of the proposition follows directly from the substitution $t=t_{0}$ at $\alpha^{+}$. 
Here, we have an example of a spherical curve $\alpha^{+}$of the curve $\gamma(t)=\left(t^{2}-t, t^{2}+\right.$ $\left.t, \sqrt{1-4 t^{3}}\right)$ in $S_{1}^{3}$. We use the Maple software to obtain the complicated expression of $\alpha^{+}$ and of the surface $\mathfrak{B}$ (that we omit here) and the Figure below. $\mathfrak{B}$ is the bifurcation set given in Section 4 .

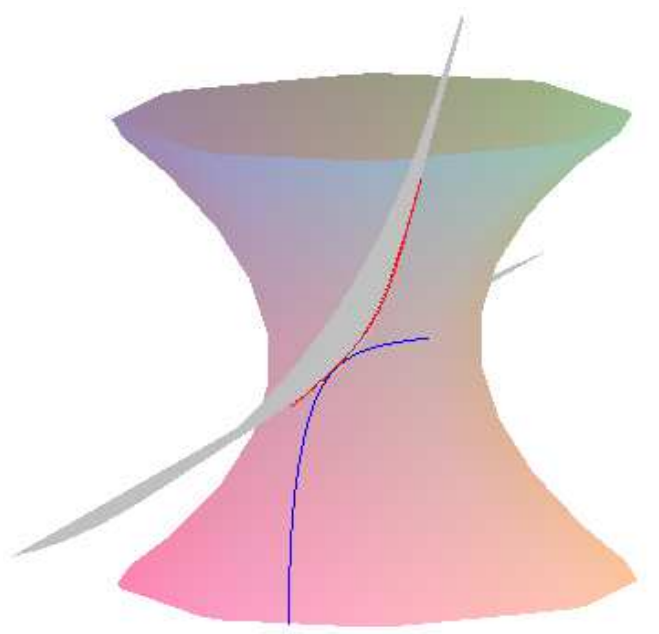

Figure 3: Example of a spherical curve $\alpha^{+}$(curve $\gamma$ is blue, $\alpha^{+}$is red and $\mathfrak{B}$ is the gray surface).

Remark 5.6 The curve $\alpha^{-}$does not intersect the curve $\gamma$, but $\alpha^{-}$intersects $-\gamma$ at the lightlike point $-\gamma\left(t_{0}\right)$. The focal set of $\gamma$ and of $-\gamma$ are the same.

\section{Focal set of curves in $S_{1}^{3}$}

In this section, we consider curves in de Sitter space $S_{1}^{3} \subset \mathbb{R}_{1}^{4}$ and we study the focal set in $S_{1}^{3}$ of these curves. To obtain the results for curves in the de Sitter space, we have Section 5 as the motivation. Let $\gamma: I \rightarrow S_{1}^{3}$ be a smooth and regular curve in $S_{1}^{3}$. In the case where the curve is spacelike or timelike, we can parametrise it by the arc length s. Thus, for the spacelike curve, we take the unit tangent vector $t(s)=\gamma^{\prime}(s)$. Suppose that $\left\langle t^{\prime}(s), t^{\prime}(s)\right\rangle \neq$ 1 , then $\left\|t^{\prime}(s)+\gamma(s)\right\| \neq 0$, and we have other unit vector $n(s)=\frac{t^{\prime}(s)+\gamma(s)}{\left\|t^{\prime}(s)+\gamma(s)\right\|}$. We also define a unit vector by $e(s)=\gamma(s) \wedge t(s) \wedge n(s)$, then we have an orthonormal basis $\{\gamma(s), t(s), n(s), e(s)\}$ of $\mathbb{R}_{1}^{4}$ along $\gamma$. The Frenet-Serret type formulae of a spacelike curve in $S_{1}^{3}$ (see [2]), are given by

$$
\left\{\begin{aligned}
\gamma^{\prime}(s) & =t(s) \\
t^{\prime}(s) & =-\gamma(s)+k_{g}(s) n(s) \\
n^{\prime}(s) & =-\delta(\gamma(s)) k_{g}(s) t(s)+\tau_{g}(s) e(s) \\
e^{\prime}(s) & =\tau_{g}(s) n(s)
\end{aligned}\right.
$$


where $\delta(\gamma(s))=\operatorname{sign}(n(s)), k_{g}(s)=\left\|t^{\prime}(s)+\gamma(s)\right\|, \tau_{g}(s)=\frac{\delta(\gamma(s))}{k_{g}^{2}(s)} \operatorname{det}\left(\gamma(s), \gamma^{\prime}(s), \gamma^{\prime \prime}(s)\right.$, $\gamma^{\prime \prime \prime}(s)$ ), and det is the determinant of the $4 \times 4$ matrix. Here $k_{g}$ is called the geodesic curvature and $\tau_{g}$ the geodesic torsion of $\gamma$ (see [2]).

Since $\left\langle t^{\prime}(s)+\gamma(s), t^{\prime}(s)+\gamma(s)\right\rangle=\left\langle t^{\prime}(s), t^{\prime}(s)\right\rangle-1$, the condition $\left\langle t^{\prime}(s), t^{\prime}(s)\right\rangle \neq 1$ is equivalent to the condition $k_{g}(s) \neq 0$.

If the curve is timelike, we take the unit tangent vector $t(s)=\gamma^{\prime}(s)$. By supposing a generic condition $\left\langle t^{\prime}(s), t^{\prime}(s)\right\rangle \neq 1$, then $\left\|t^{\prime}(s)-\gamma(s)\right\| \neq 0$, and we have other unit vector $n(s)=\frac{t^{\prime}(s)-\gamma(s)}{\left\|t^{\prime}(s)-\gamma(s)\right\|}$. We also define an unit vector by $e(s)=\gamma(s) \wedge t(s) \wedge n(s)$, then we have an orthonormal basis $\{\gamma(s), t(s), n(s), e(s)\}$ of $\mathbb{R}_{1}^{4}$ along $\gamma$. Thus, the Frenet-Serret type formulae of a timelike curve in $S_{1}^{3}$ are given by

$$
\left\{\begin{aligned}
\gamma^{\prime}(s) & =t(s) \\
t^{\prime}(s) & =\gamma(s)+k_{h}(s) n(s) \\
n^{\prime}(s) & =k_{h}(s) t(s)+\tau_{h}(s) e(s) \\
e^{\prime}(s) & =-\tau_{h}(s) n(s)
\end{aligned}\right.
$$

where $k_{h}(s)=\left\|t^{\prime}(s)-\gamma(s)\right\|$ and $\tau_{g}(s)=-\frac{1}{k_{h}^{2}(s)} \operatorname{det}\left(\gamma(s), \gamma^{\prime}(s), \gamma^{\prime \prime}(s), \gamma^{\prime \prime \prime}(s)\right)$. Here $k_{h}$ is called the hyperbolic curvature and $\tau_{h}$ the hyperbolic torsion of $\gamma$ (see [6]).

Since $\left\langle t^{\prime}(s)-\gamma(s), t^{\prime}(s)-\gamma(s)\right\rangle=\left\langle t^{\prime}(s), t^{\prime}(s)\right\rangle-1$, the condition $\left\langle t^{\prime}(s), t^{\prime}(s)\right\rangle \neq 1$ is equivalent to the condition $k_{h}(s) \neq 0$.

Consider the family of distance squared functions, $f: I \times S_{1}^{3} \rightarrow \mathbb{R}$, on $\gamma$

$$
f(s, v)=\langle\gamma(s)-v, \gamma(s)-v\rangle
$$

where $f_{v}(s)=f(s, v)$, for some $v \in S_{1}^{3}$ fixed. Observe that since $v \in S_{1}^{3}$, then $-\frac{1}{2} f_{v}(s)=$ $\langle\gamma(s), v\rangle-1$ and the singularities of the distance squared function and the height function are the same.

The spherical bifurcation set of $f$ is given by

$$
\mathfrak{B i f}(f)=\left\{v \in S_{1}^{3} \mid f_{v}^{\prime}(s)=f_{v}^{\prime \prime}(s)=0 \text { at }(s, v) \text { for some } s\right\},
$$

i.e., the directions where the singularity of $f$ at $s$ is $A_{\geq 2}$. This is also defined for the lightlike points of $\gamma$.

The spherical focal surface of $\gamma$ coincides with the spherical bifurcation set of $f$. Furthermore, for curves in $S_{1}^{3} \subset \mathbb{R}_{1}^{4}$ the spherical focal surface is the intersection of the focal hypersurface in $\mathbb{R}_{1}^{4}$ with the de Sitter space $S_{1}^{3}$.

For a spacelike curve $\gamma$ parametrised by the arc length with $k_{g}(s) \neq 0$, we have that the spherical focal surface of $\gamma$ is given by

$$
\mathfrak{B}^{ \pm}(s, \mu)=\mu \gamma(s)+\frac{\mu}{\delta(\gamma(s)) k_{g}(s)} n(s) \pm \frac{\sqrt{-\delta(\gamma(s)) k_{g}^{2}(s)+\delta(\gamma(s)) \mu^{2}\left(k_{g}^{2}(s)+\delta(\gamma(s))\right.}}{k_{g}(s)} e(s)
$$


with $\mu \in \mathbb{R}$. The $g$-spherical cuspidal curve is given by $\mathfrak{B}^{ \pm}(s, \mu(s))=\mathfrak{B}^{ \pm}(s)$, where

$$
\mu(s)=\frac{ \pm \tau_{g}(s) k_{g}^{2}(s)}{\sqrt{\tau_{g}^{2}(s) k_{g}^{4}(s)-k_{g}^{\prime 2}(s) \delta(\gamma(s))-\tau_{g}^{2}(s) k_{g}^{2}(s) \delta(\gamma(s))}} .
$$

For a timelike curve $\gamma$ parametrised by the arc length with $k_{h}(s) \neq 0$, the spherical focal surface is given by

$$
\mathfrak{B}^{ \pm}(s, \mu)=\mu \gamma(s)-\frac{\mu}{k_{h}(s)} n(s) \pm \frac{\sqrt{k_{h}^{2}(s)-\mu^{2}\left(k_{h}^{2}(s)+1\right)}}{k_{h}(s)} e(s),
$$

with $\mu \in \mathbb{R}$. The $h$-spherical cuspidal curve is given by $\mathfrak{B}^{ \pm}(s, \mu(s))=\mathfrak{B}^{ \pm}(s)$, where

$$
\mu(s)=\frac{ \pm \tau_{h}(s) k_{h}^{2}(s)}{\sqrt{\tau_{h}^{2}(s) k_{h}^{4}(s)+k_{h}^{2}(s)+\tau_{h}^{2}(s) k_{h}^{2}(s)}} .
$$

Remark 6.1 The spherical focal surface of a spacelike curve is defined if $-\delta(\gamma(s)) k_{g}^{2}(s)+$ $\delta(\gamma(s)) \mu^{2}\left(k_{g}^{2}(s)+\delta(\gamma(s)) \geq 0\right.$. As $k_{g}(s) \neq 0$, we have that $n(s)$ is spacelike or timelike. In the case where $n(s)$ is spacelike, the spherical focal surface is defined for

$$
\mu \leq-\frac{k_{g}(s)}{\sqrt{k_{g}^{2}(s)+1}} \text { or } \mu \geq \frac{k_{g}(s)}{\sqrt{k_{g}^{2}(s)+1}},
$$

otherwise in the case that $n(s)$ is timelike, the spherical focal surface is defined for

$$
-\frac{k_{g}(s)}{\sqrt{k_{g}^{2}(s)-1}} \leq \mu \leq \frac{k_{g}(s)}{\sqrt{k_{g}^{2}(s)-1}} .
$$

The spherical focal surface of a timelike curve is defined if

$$
-\frac{k_{h}(s)}{\sqrt{k_{h}^{2}(s)+1}} \leq \mu \leq \frac{k_{h}(s)}{\sqrt{k_{h}^{2}(s)+1}} .
$$

Furthermore, in both cases where $\mathfrak{B}^{+}(s, \mu)$ and $\mathfrak{B}^{-}(s, \mu)$ are symmetric, then we study only $\mathfrak{B}^{+}(s, \mu)$.

We prove in the next results that the tangent plane of the spherical focal surface of a spacelike curve (respectively, timelike) is not defined at the points of the $g$-spherical cuspidal curve (respectively, of the $h$-spherical cuspidal curve). Furthermore, away from these curves we analyse the metric structure of the spherical focal surface in each case.

Away from the $g$-spherical cuspidal curve,

$$
\mu(s) \neq \frac{ \pm \tau_{g}(s) k_{g}^{2}(s)}{\sqrt{\tau_{g}^{2}(s) k_{g}^{4}(s)-k_{g}^{\prime 2}(s) \delta(\gamma(s))-\tau_{g}^{2}(s) k_{g}^{2}(s) \delta(\gamma(s))}},
$$


or away from the $h$-spherical cuspidal curve, $\mu(s) \neq \frac{ \pm \tau_{h}(s) k_{h}^{2}(s)}{\sqrt{\tau_{h}^{2}(s) k_{h}^{4}(s)+k_{h}^{\prime 2}(s)+\tau_{h}^{2}(s) k_{h}^{2}(s)}}$, $v=\lambda_{1} \mathfrak{B}_{s}^{+}+\lambda_{2} \mathfrak{B}_{\mu}^{+}$, with $\lambda_{1}, \lambda_{2} \in \mathbb{R}$, are the vectors of the tangent plane of the spherical focal surface at $\mathfrak{B}^{+}(s, \mu)$ and $\langle v, v\rangle=\lambda_{1}^{2}\left\langle\mathfrak{B}_{s}^{+}, \mathfrak{B}_{s}^{+}\right\rangle+2 \lambda_{1} \lambda_{2}\left\langle\mathfrak{B}_{s}^{+}, \mathfrak{B}_{\mu}^{+}\right\rangle+\lambda_{2}^{2}\left\langle\mathfrak{B}_{\mu}^{+}, \mathfrak{B}_{\mu}^{+}\right\rangle$, by using the respective parametrisation of $\mathfrak{B}(s, \mu)$ for spacelike or timelike $\gamma$.

Proposition 6.2 Let $\gamma$ be a spacelike curve.

(a) The tangent plane of the spherical focal surface of $\gamma$ is not defined on the g-spherical cuspidal curve.

(b) Away from the g-spherical cuspidal curve, the spherical focal surface of $\gamma$ is timelike.

Proof (a) Considering a spacelike curve, the tangent plane at the points of the spherical focal surface is generated by the vectors

$$
\begin{aligned}
\mathfrak{B}_{s}^{+}(s, \mu) & =\left(\frac{\left.-\mu k_{g}^{\prime}(s)+\delta(\gamma(s)) \tau_{g}(s) k_{g}(s) \sqrt{-\delta(\gamma(s)) k_{g}^{2}(s)+\delta(\gamma(s)) \mu^{2}\left(k_{g}^{2}(s)+\delta(\gamma(s))\right.}\right)}{\delta k_{g}^{2}(s)}\right) n(s) \\
& +\left(\frac{\left.\mu \tau_{g}(s) k_{g}(s) \sqrt{-\delta(\gamma(s)) k_{g}^{2}(s)+\delta(\gamma(s)) \mu^{2}\left(k_{g}^{2}(s)+\delta(\gamma(s))\right.}\right)-\delta\left(\gamma(s) \mu^{2} k_{g}^{\prime}(s)\right)}{\left.\delta(\gamma(s)) k_{g}^{2}(s) \sqrt{-\delta(\gamma(s)) k_{g}^{2}(s)+\delta(\gamma(s)) \mu^{2}\left(k_{g}^{2}(s)+\delta(\gamma(s))\right.}\right)}\right) e(s)
\end{aligned}
$$

and

$\mathfrak{B}_{\mu}^{+}(s, \mu)=\gamma(s)+\frac{1}{\delta(\gamma(s)) k_{g}(s)} n(s)+\left(\frac{\delta(\gamma(s)) \mu\left(k_{g}^{2}(s)+\delta(\gamma(s))\right)}{k_{g}(s) \sqrt{-\delta(\gamma(s)) k_{g}^{2}(s)+\delta(\gamma(s)) \mu^{2}\left(k_{g}^{2}(s)+\delta(\gamma(s))\right.}}\right) e(s)$.

The vectors $\mathfrak{B}_{s}^{+}$and $\mathfrak{B}_{\mu}^{+}$are linearly dependent if and only if

$$
\mu(s)=\frac{ \pm \tau_{g}(s) k_{g}^{2}(s)}{\sqrt{\tau_{g}^{2}(s) k_{g}^{4}(s)-k_{g}^{\prime 2}(s) \delta(\gamma(s))-\tau_{g}^{2}(s) k_{g}^{2}(s) \delta(\gamma(s))}}
$$

that is precisely where the tangent plane is not defined and furthermore is where $f_{v}$ has singularities of type $A_{\geq 3}$, that is the g-spherical cuspidal curve.

(b) Let $\gamma$ be a spacelike curve. Let us suppose that $n(s)$ is spacelike and $e(s)$ is timelike, thus making $\langle v, v\rangle=0$, and thinking of this equation as a quadratic equation, then

$$
\Delta=4 \lambda_{2}^{2} \frac{\left(\tau_{g} k_{g} \sqrt{-k_{g}^{2}+\mu^{2}\left(k_{g}^{2}+1\right)}-\mu k_{g}^{\prime}\right)^{2}}{k_{g}^{2}\left(-k_{g}^{2}+\mu^{2}\left(k_{g}^{2}+1\right)\right)} .
$$

The tangent plane generated by $\mathfrak{B}_{s}^{+}$and $\mathfrak{B}_{\mu}^{+}$can be lightlike, if $\Delta=0$. As we are supposing $\tau_{g}(s) k_{g}(s) \sqrt{-k_{g}^{2}(s)+\mu^{2}\left(k_{g}^{2}(s)+1\right)}-\mu k_{g}^{\prime}(s) \neq 0$, for $\mathfrak{B}_{s}^{+}$and $\mathfrak{B}_{\mu}^{+}$be linearly 
independent, then we have $\Delta=0$ if and only if $\lambda_{2}=0$, that is, if $\mathfrak{B}_{s}^{+}$is lightlike. But, $\mathfrak{B}_{s}^{+}(s, \pm 1)$ are the only lightlike vectors. Besides $\mathfrak{B}_{\mu}^{+}(s, \pm 1)$ are timelike vectors, i.e., the tangent planes at the points $(s, \pm 1)$ are timelike. Since, we have $\Delta>0$ at the others points, thus the spherical focal surface is timelike.

Proposition 6.3 Let $\gamma$ be a timelike curve.

(a) The tangent plane of the spherical focal surface of $\gamma$ is not defined on the $h$-spherical cuspidal curve.

(b) Away from the h-spherical cuspidal curve, the spherical focal surface of $\gamma$ is spacelike.

Proof The proofs are analogous to the proofs of Proposition 6.2. In case (b), we observe that $\mathfrak{B}_{s}^{+}(s, \pm 1)$ are not defined, then we prove that $\Delta<0$ for the equation $\langle v, v\rangle=0$.

Now our aim is to find a general expression for the $\mathfrak{B i f}(f)$ to know what is happening to the spherical focal surface near a lightlike point of $\gamma$. For this, consider the curve $\gamma$ not parametrised by the arc length and a vector $N(t)$ such that $\gamma(t), N(t)$, and $E(t)=$ $\gamma(t) \wedge \gamma^{\prime}(t) \wedge N(t)$ generate the normal hyperplane to the vector $\gamma^{\prime}(t)$. By definition, we have that $\mathfrak{B i f}(f)$ of $\gamma$ is a spherical surface of $\gamma$ given by

$$
\mathfrak{B}^{ \pm}(t, \mu)=\mu \gamma(t)+\beta(t, \mu) N(t)+\lambda(t, \mu) E(t),
$$

where $\beta$ and $\lambda$ satisfies the equations below:

$$
\lambda(t, \mu)=\frac{\mu\left\langle\gamma^{\prime}(t), \gamma^{\prime}(t)\right\rangle-\beta\left\langle\gamma^{\prime \prime}(t), N(t)\right\rangle}{\left\langle\gamma^{\prime \prime}(t), E(t)\right\rangle} \quad \text { and }
$$

$\beta(t, \mu)$ is equal to

$$
\left(\frac{\mu\left\langle\gamma^{\prime}, \gamma^{\prime}\right\rangle\left\langle\gamma^{\prime \prime}, N\right\rangle\langle E, E\rangle \pm \sqrt{\left\langle\gamma^{\prime \prime}, N\right\rangle^{2}\langle E, E\rangle\left\langle\gamma^{\prime \prime}, E\right\rangle^{2}\left(1-\mu^{2}\right)+\langle N, N\rangle\left\langle\gamma^{\prime \prime}, E\right\rangle^{4}\left(1-\mu^{2}\right)-\langle N, N\rangle\left\langle\gamma^{\prime \prime}, E\right\rangle^{2}\left\langle\gamma^{\prime}, \gamma^{\prime}\right\rangle^{2}\langle E, E\rangle \mu^{2}}}{\left\langle\gamma^{\prime \prime}, E\right\rangle^{2}\langle N, N\rangle^{2}+\langle E, E\rangle\left\langle\gamma^{\prime \prime}, N\right\rangle^{2}}\right)
$$

where $\mu$ is real number such that the root of $\beta$ is defined.

Remark 6.4 The spherical surface $\mathfrak{B}^{ \pm}$is well defined near a lightlike point $\gamma\left(t_{0}\right)$. Let $R(t, \mu)=A(t) \mu^{2}+B(t)$, the term inside the squared root of the above $\beta$, where

$$
\begin{aligned}
A(t) & =\left(-\left\langle\gamma^{\prime \prime}, N\right\rangle^{2}\langle E, E\rangle\left\langle\gamma^{\prime \prime}, E\right\rangle^{2}-\langle N, N\rangle\left\langle\gamma^{\prime \prime}, E\right\rangle^{4}-\langle N, N\rangle\left\langle\gamma^{\prime \prime}, E\right\rangle^{2}\left\langle\gamma^{\prime}, \gamma^{\prime}\right\rangle^{2}\langle E, E\rangle\right)(t) \\
\text { and } \quad B(t) & =\left(\left\langle\gamma^{\prime \prime}, E\right\rangle^{2}\left\langle\gamma^{\prime \prime}, N\right\rangle^{2}\langle E, E\rangle+\langle N, N\rangle\left\langle\gamma^{\prime \prime}, E\right\rangle^{4}\right)(t) .
\end{aligned}
$$

Then for the spherical surface $\mathfrak{B}^{ \pm}$be defined, we must have $R(t, \mu) \geq 0$. Making the calculations at the lightlike point we have $A\left(t_{0}\right)<0$ and $B\left(t_{0}\right)>0$ (these are equal in module) and in this case the spherical surface $\mathfrak{B}^{ \pm}$is defined when $R\left(t_{0}, \mu\right)=A\left(t_{0}\right) \mu^{2}+B\left(t_{0}\right) \geq 0$, i.e., $-1 \leq \mu \leq 1$. Thus, for continuity, there is a neighborhood near the $\left(t_{0}, \mu\right)$ such that $R(t, \mu) \geq 0$.

Proposition 6.5 The spherical surface $\mathfrak{B}^{ \pm}$intersects the curve $\gamma$ at lightlike points and the tangent planes to $\mathfrak{B}^{ \pm}$are not defined at these points. 
Proof Let $\gamma\left(t_{0}\right)$ be a lightlike point of $\gamma$. Analysing the expression of the spherical surface $\mathfrak{B}^{ \pm}$we have $\mathfrak{B}^{ \pm}\left(t_{0}, 1\right)=\gamma\left(t_{0}\right)$, because $\beta\left(t_{0}, 1\right)=0$ and $\lambda\left(t_{0}, 1\right)=0$. Since $R\left(t_{0}, 1\right)=0$, then the tangent planes to the spherical surface at $\mathfrak{B}^{ \pm}\left(t_{0}, 1\right)$ are not defined.

We observe that $\mathfrak{B}^{+}\left(t_{0},-1\right)=\mathfrak{B}^{-}\left(t_{0},-1\right)=-\gamma\left(t_{0}\right)$ and the bifurcation set of $\gamma$ and $-\gamma$ are the same. Furthermore $R\left(t_{0},-1\right)=0$, then the tangent planes to the spherical surface $\mathfrak{B}^{ \pm}$also are not defined at these points. Then, we have the next result.

Proposition 6.6 The $L D$ set of the spherical surface $\mathfrak{B}^{ \pm}$are the curves $\mathfrak{B}^{ \pm}\left(t_{0}, \mu\right),-1<$ $\mu<1$.

Proof The tangent planes at $\mathfrak{B}^{ \pm}\left(t_{0}, \mu\right)$ exist for $-1<\mu<1$. The proof follows observing that the spherical surface $\mathfrak{B}^{ \pm}$is the union of the spherical focal surface of the spacelike and timelike part of $\gamma$, with the curves $\mathfrak{B}^{ \pm}\left(t_{0}, \mu\right),-1<\mu<1$.

\section{References}

[1] Bruce, J. W., And Giblin, P. J. Curves and Singularities: a geometrical introduction to singularity theory. Cambridge university press, 1992.

[2] Fusho, T., And Izumiya, S. Lightlike surfaces of spacelike curves in de Sitter 3-space. J. Geom. 88, 1-2 (2008), 19-29.

[3] Izumiya, S. Generating families of developable surfaces in $\mathbb{R}^{3}$. Hokkaido University preprint series in mathematics 512, 2001.

[4] Izumiya, S., Kikuchi, M., and Takahashi, M. Global properties of spacelike curves in Minkowski 3-space. J. Knot Theory Ramifications 15, 7 (2006), 869-881.

[5] Izumiya, S., Pei, D., And Sano, T. Singularities of hyperbolic gauss maps. Proceedings of the London mathematical Society 86, 2 (2003), 485-512.

[6] Izumiya, S., Pei, D., And Sano, T. Horospherical surfaces of curves in hyperbolic space. Publ. Math. Debrecen 64, 1-2 (2004), 1-13.

[7] Izumiya, S., Pei, D. H., Sano, T., and Torit, E. Evolutes of hyperbolic plane curves. Acta Math. Sin. (Engl. Ser.) 20, 3 (2004), 543-550.

[8] Izumiya, S., and Takiyama, A. A timelike surface in Minkowski 3-space which contains pseudocircles. Proc. Edinburgh Math. Soc. (2) 40, 1 (1997), 127-136.

[9] Pei, D., And Sano, T. The focal developable and the binormal indicatrix of a nonlightlike curve in Minkowski 3-space. Tokyo J. Math. 23, 1 (2000), 211-225.

[10] Pelletier, F. Quelques propriétés géométriques des variétés pseudo-riemanniennes singulières. In Annales de la faculté des sciences de Toulouse (1995), vol. 4, Université Paul Sabatier, pp. 87-199. 
[11] Ratcliffe, J. Foundations of hyperbolic manifolds, vol. 149. Springer, 2006.

[12] Saloom, A., And TARI, F. Curves in the Minkowski plane and their contact with pseudo-circles. Geom. Dedicata 159 (2012), 109-124.

[13] Steller, M. A gauss-bonnet formula for metrics with varying signature. Zeitschrift für Analysis und ihre Anwendungen 25, 2 (2006), 143-162.

[14] TARI, F. Caustics of surfaces in the Minkowski 3-space. Q. J. Math. 63, 1 (2012), 189-209.

Ana Claudia Nabarro, Andrea de Jesus Sacramento, Departamento de Matemática, ICMC Universidade de São Paulo, Campus de São Carlos, Caixa Postal 668, CEP 13560-970, São Carlos-SP, Brazil

e-mail:anaclana@icmc.usp.br

e-mail:andreajs@icmc.usp.br 